\title{
De la contraception en général et de la contraception masculine en particulier
}

Depuis la commercialisation de la pilule ostro-progestative, à la fin des années 1960, la contraception féminine a fait d'énormes progrès.

Malgré l'opposition habituelle des religions, idéologies et des politiques natalistes, la demande féminine de contrôle des naissances, sans doute aussi vieille que le monde [1], entretient dans les pays développés un marché dont l'ampleur a stimulé l'implication de l'industrie pharmaceutique. Celle-ci, cependant, montrerait des réticences croissantes à développer de nouvelles recherches alors qu'existent des produits fiables et peu coûteux et qu'apparaissent des risques juridico-économiques liés aux effets secondaires éventuels des nouvelles méthodes [2]. La production des contraceptifs oraux classiques semble stagner, en raison peut-être de la saturation du marché occidental et de la non-solvabilité des femmes du tiers-monde.

L'histoire de la contraception masculine est pauvre en événements. La vieille pratique du coït interrompu est économique, peu fiable et inconfortable. Le préservatif, avant tout conçu comme protection contre les maladies sexuellement transmissibles, n'est devenu moyen de contraception qu'au XXème siècle [3]. La vasectomie reste une méthode de stérilisation plutôt que de contraception en raison de son incertaine réversibilité. La mise au point d'une contraception hormonale masculine a débuté il y a une vingtaine d'années [4] dans un climat de suspicion encore persistant.

Dans ce numéro d'Andrologie nous proposons aux lecteurs trois articles de mise au point sur les approches modernes de la contraception masculine hormonale, thermique et immunologique.

La contraception masculine est évidemment en retard sur la contraception féminine. Les raisons en sont diverses. La physiologie sexuelle masculine est plus mal connue que la féminine et certaines particularités de cette physiologie rendent plus difficile le contrôle hormonal de la spermatogénèse que celui de l'ovulation. Mais, surtout, le contrôle de la conception a toujours été avant tout une affaire de femmes directement et profondément impliquées par ses conséquences. Il ne semble pas, à la veille de l'an 2000 que les hommes demandeurs de contraception masculine soient très nombreux. Les représentations de la fertilité et de la virilité forment encore un complexe mal dissociable, composante majeure de l'identité masculine. Les motivations des scientifiques désireux d'inventer de nouvelles méthodes ne sont pas celles des utilisateurs dont le désir éventuel d'assumer des responsabilités est fortement contrebalancé par la peur de supporter les conséquences réelles ou imaginaires des moyens employés. Le phénomène est suffisamment évident pour influencer une économie de marché manifestement plus disposée à investir dans des 
programmes de recherche et de commercialisation de substances, si possible actives par voie orale, capables de restaurer ou stimuler l'érection.

Il reste qu'à défaut de déboucher sur des applications pratiques de grande diffusion, les recherches sur la contraception masculine apportent et apporteront une importante contribution à la connaissance de la physiologie.

En conclusion de ces quelques réflexions nous voudrions ébaucher un parrallèle troublant. On peut considérer que les hommes manifestent d'autant moins d'intérêt pour la contraception masculine qu'ils estiment que le contrôle des naissances est suffisamment bien assuré par les femmes elles-mêmes. Le succès de l'ICSI n'est-il pas de la même façon démobilisateur en ce qui concerne la recherche et la demande de traitements de l'infertilité masculine? N'est-il pas plus confortable pour les hommes de voir leurs problèmes pris en charge par leurs partenaires?

J.C. CZYBA

\section{REFERENCES}

1. MC LAREn A. : Histoire de la contraception, de l'Antiquité à nos jours. Noêsis, Paris, 1996.

2. SERviCE R.F. : Panel wants to break R and D barrier. Science, 1996, 272, 1258.

3. Androutsos G., Marketos S.P. : L'Histoire du préservatif. Andrologie, 1994, 4, 492-504.

4. GUERIN J.F. : La contraception masculine. Simep, Lyon-Villeurbanne, 1984. 